\title{
Single camera three component planar velocity measurements using two frequency Planar Doppler Velocimetry (2v-PDV)
}

\author{
T.O.H. Charrett and R.P. Tatam \\ Optical Sensors Group, Centre for Photonics and Optical Engineering, School of \\ Engineering, Cranfield University, Cranfield, Bedford. MK43 0AL, UK \\ E-mail: r.p.tatam@cranfield.ac.uk
}

\begin{abstract}
Two frequency Planar Doppler Velocimetry (2v-PDV) is used in conjunction with imaging fibre bundles to make time-averaged three component velocity measurements using a single CCD camera and iodine cell. In this scheme the flow is sequentially illuminated with two different frequencies of laser light, which are separated by approximately $700 \mathrm{MHz}$ to $1 \mathrm{GHz}$. Images are captured for each illumination frequency on a CCD camera viewing through an iodine absorption filter. These images are then used to produce a normalised transmission image that can be processed as in normal PDV. Three component measurements are made possible by porting separate views to a single CCD camera using the imaging fibre bundles. Results are presented here for a $2 v$-PDV system demonstrated making time-averaged velocity measurements on a rotating disc and a seeded air jet.
\end{abstract}

Keywords. Planar Doppler Velocimetry, flow measurement, acousto-optic frequency switching, coherent fibre bundles

\section{Introduction}

The non-intrusive measurement of flow using laser methods has proven to be invaluable as a diagnostic tool for many years both for the designer and also for those interested in fundamental investigations of fluid mechanics. Original optical systems, laser Doppler anemometry (LDA) and laser transit anemometry (LTA), measure velocities at a point within the flow with high temporal resolution. However, the time taken to map flow fields, for example in large wind tunnel facilities, is very costly and often precludes their use. In addition there is increasing interest in techniques able to provide information simultaneously from different regions of the flow thus enabling spatial features of the flow to be measured. The development of planar techniques has helped to address this issue. In planar flow measurement a region of the flow is illuminated with a laser beam conditioned to form a light sheet in the region of interest. The light scattered from particles entrained in the flow is imaged onto two-dimensional detector arrays, usually CCDs.

The most developed planar flow measurement method is particle image velocimetry (PIV) in which two images of the flow are collected with a short time difference between them [1]. Correlation techniques are then used to calculate the distance and direction a particle has moved. A single viewing direction and laser sheet results in the measurement of two velocity components in the plane of the sheet. The out of plane velocity component is more difficult to extract but techniques based on stereoscopic viewing have shown some success. PIV requires a well-controlled seeding density and particle size and is limited to reasonably small flow field sizes.

In contrast the more recent technique of Planar Doppler Velocimetry (PDV)[2-5], also know as Doppler Global Velocimetry (DGV), is capable of operating with a range of seeding densities, large flow fields and high speed flows $(>100 \mathrm{~m} / \mathrm{s})$. Three components of velocity can be measured instantaneously or timeaveraged depending on the laser source and image processing methods used. PDV was originally implemented using conventional optical components, but more recently the addition of optical fibres to the instrumentation has added considerable flexibility and functionality to the technique[6-11]. 
PDV relies upon measuring the Doppler frequency shift of light scattered from particles entrained in the flow. The technique allows the measurement of the three-dimensional flow velocities over many points in the plane, quickly and non-intrusively.

A single observation direction can measure a single component of velocity, as shown in figure 1, and multiple viewing, or illumination, directions are required for multiple velocity component measurements. This would usually require multiple imaging systems, however the use of imaging fibre bundles[9] allows several observation directions, and thus velocity components, to be measured simultaneously by spatially multiplexing on to a single CCD camera.

The optical frequency shift, $\Delta v$, is given by the Doppler equation:

$$
\Delta v=\frac{v(\hat{\boldsymbol{o}}-\hat{\boldsymbol{i}}) \cdot V}{c}
$$

where $\boldsymbol{v}$ is the optical frequency, $\hat{\boldsymbol{o}}$ and $\hat{\boldsymbol{i}}$ are unit vectors in the observation and illumination directions respectively, $\boldsymbol{V}$ is the velocity vector and $\boldsymbol{c}$ is the free space speed of light.

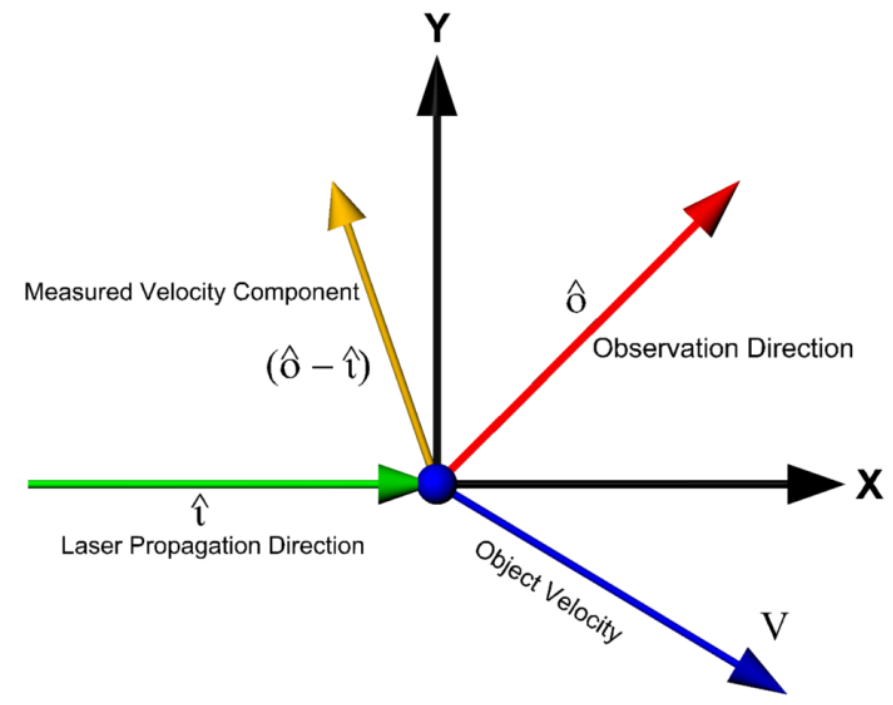

Figure 1. The relationship of laser illumination direction and observation direction to the measured velocity component determined from the Doppler equation.

The optical frequency of light scattered from each particle in the seeded flow experiences a Doppler shift, which is linearly related to the velocity of the particle. In PDV, a region of the illuminated flow is imaged, through a glass cell usually containing iodine vapour, onto the active area of a CCD camera. Iodine has numerous narrow absorption lines over a large part of the visible spectrum[12] and the laser frequency is chosen to coincide with one such line. Hence the optical intensity at any position in the camera image is a function of the Doppler shift experienced at the corresponding position in the flow, via the frequencydependent iodine absorption.

The intensity over a PDV image is also affected by the intensity profile of the illuminating laser sheet (typically Gaussian), spatial variations of the seeding density within the flow, and diffraction fringes caused by imperfections in the optical surfaces. These variations are generally of similar amplitude to those resulting from absorption in the iodine cell, and can obscure the information about flow velocity that is contained within the camera image. It is therefore usual to amplitude-divide the image beam onto two cameras, figure 2; from one of the two imaging paths the iodine cell is omitted, and the resulting image acts as a reference to normalize the signal image carrying the velocity information.

\section{Two frequency Planar Doppler Velocimetry (2v-PDV)}


In the two-frequency Planar Doppler Velocimetry (2v-PDV)[10] technique the signal and reference images are acquired sequentially, on the same CCD camera, figure 3, by the use of two illumination frequencies. There are two approaches to the positioning of the illuminations frequencies relative to the iodine absorption line.

In the first a signal and reference image are captured similar to conventional PDV. The first illumination frequency is tuned just off the low frequency side of an absorption line to lie in a 100\% transmission region of the iodine transfer function (figure 4, point A). A reference image is then acquired. The second illumination frequency is positioned at approximately midway $(50 \%)$ on the iodine cell transfer function (figure 4, point B), and a signal image is acquired. The two images can then be processed as in conventional PDV, by dividing the two images to produce a normalised transmission image that is then used to calculate the frequency, Doppler shift and finally the velocity.

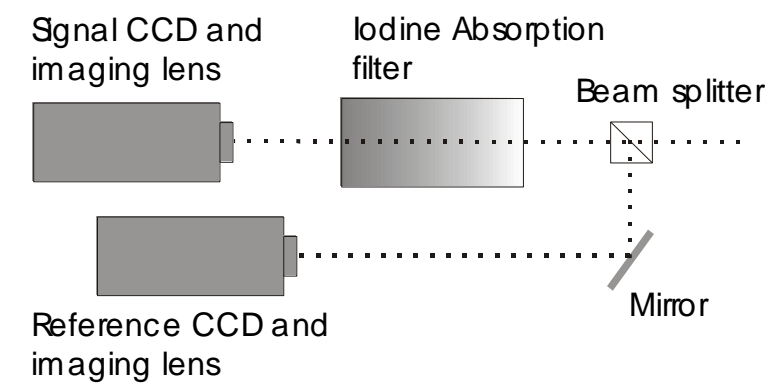

Figure 2. Conventional two CCD camera, PDV imaging head arrangement.

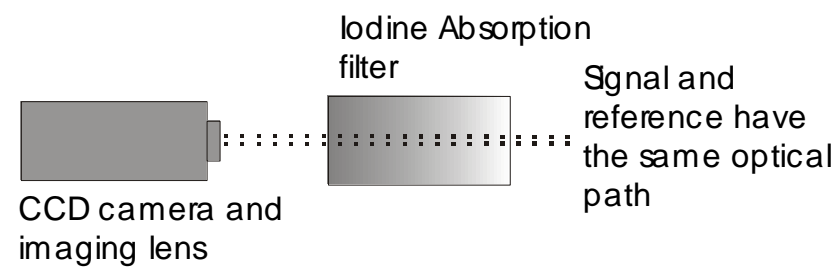

Figure 3. Two frequency PDV, single CCD camera, imaging head.

The second approach increases the sensitivity of the system by tuning the two frequencies to the positions shown as B and C in figure 4. With one source tuned onto the falling slope and the other on the rising slope, a constant Doppler shift will result in the further attenuation of one image to a lower signal level and the rise in the signal level in the other image. Dividing the difference of the images by the sum, and taking into account any difference in the gradients will give a result that has approximately double the sensitivity of the current PDV methods.

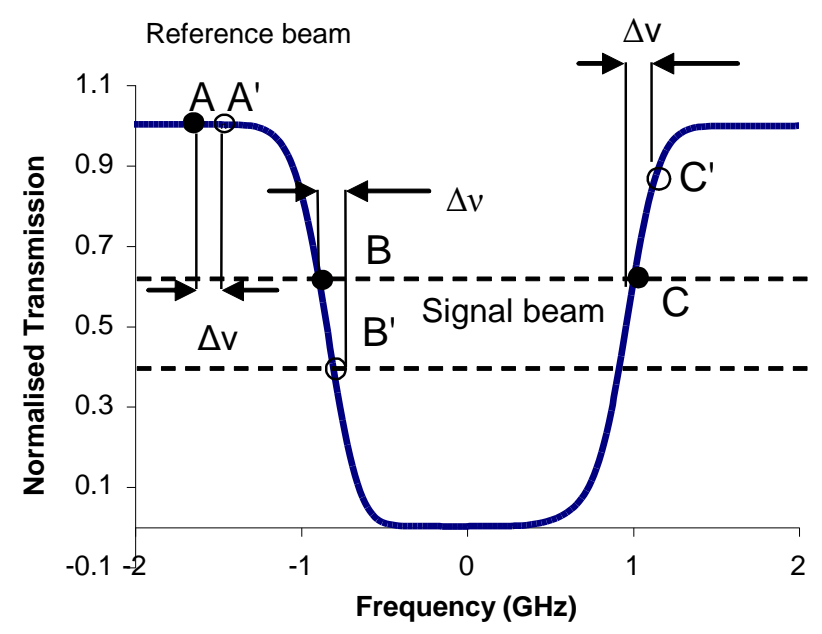

Figure 4. Relative positions of the laser frequency, and the shifted frequency on a typical absorption feature for $2 v$-PDV (A and B/C denote the position of the illumination frequencies and $\mathrm{A}^{\prime}$ and $\mathrm{B}^{\prime} / \mathrm{C}^{\prime}$ the Doppler shifted frequency) 
If the initial (un-shifted) transmission of each frequency is located on the linear portion of each slope, and the expected Doppler shifts will not cause the scattered frequency to fall outside of the linear region then the normalisation can be performed using the following analysis. Here each slope is assumed to have a constant gradient.

If the flow is illuminated using two frequencies of laser light, which are tuned to opposite sides of the iodine absorption line, as shown in figure 4, (points B and C), then the Doppler shift expressed in terms of the change in transmission (for the first beam), $\Delta \boldsymbol{T}_{1}$, can be obtained.

If the intensity of the light scattered from the flow at any point is given by, $\boldsymbol{I}_{s}$, then this can be assumed to be the same under both illuminations, if the time interval between them is short and the seeding conditions remain constant, as the difference in the Mie scattering for the closely spaced frequencies will be negligible. There is no discernable differences in Mie scattering until the wavelength difference is $\sim 0.5 \mathrm{~nm}$, here we use a difference of $\sim 0.001 \mathrm{~nm}$.

The intensity recorded at a pixel on the CCD will depend upon this scattered intensity and the absorption that occurs in the iodine cell, which is dependant upon the frequency of the illuminating light.

Under the illumination of the first frequency, $\boldsymbol{v}_{\boldsymbol{l}}$, the recorded intensity, $\boldsymbol{I}_{\boldsymbol{I}}$, will be given by:

$$
I_{1}=\left(T_{1}+\Delta T_{1} m_{1}\right) I_{s}
$$

Similarly under the illumination of the second frequency, $\boldsymbol{v}_{2}$, the recorded intensity, $\boldsymbol{I}_{2}$, will be given by:

$$
I_{2}=\left(T_{2}-\Delta T_{1} m_{2}\right) I_{s}
$$

Where:

$I_{1}, I_{2}$

$I_{s}$

$T_{1}, T_{2}$

$\Delta T_{1}$

$M_{1}, M_{2}$

$m_{1}=\left|\frac{M_{1}}{M_{1}}\right|=1$

$m_{2}=\left|\frac{M_{2}}{M_{1}}\right|$
Recorded intensity at any pixel on the CCD under the illumination of $\boldsymbol{v}_{\boldsymbol{l}}$ and $\boldsymbol{v}_{\boldsymbol{2}}$ The intensity of the light scattered from any point in the flow.

Initial (un-shifted) transmission ratios of the illumination frequencies, $\boldsymbol{v}_{\boldsymbol{l}}$ and $\boldsymbol{v}_{2}$ Change in transmission ratio (on the negative gradient slope of the iodine absorption line.)

Gradients functions of the two sides of the iodine absorption line

Normalised gradient of the negative gradient side of the iodine absorption line.

By rearranging and equating (2) and (3) the change in transmission is given by:

$$
\begin{gathered}
I_{1}\left(T_{2}-\Delta T_{1} m_{2}\right)=I_{2}\left(T_{1}+\Delta T_{1} m_{1}\right) \\
\Delta T_{1}=\frac{\left(I_{1} T_{2}-I_{2} T_{1}\right)}{\left(m_{1} I_{2}+m_{2} I_{1}\right)}
\end{gathered}
$$

This shift can then be added to the initial transmission ratio, $T_{1}$, of the first illumination frequency to find the normalised transmission, $\boldsymbol{R}$, as found in conventional PDV using a signal divided by a reference. Alternatively the Doppler frequency shift can be calculated directly from $\Delta \boldsymbol{T}_{1}$ using the gradient of the first (negative gradient) slope of the absorption line.

$$
R=T_{1}+\Delta T_{1}
$$


However as the gradient of each slope is not constant, a look-up table can be used to perform the normalisation. The value of the ratio $\boldsymbol{I}_{1} / \boldsymbol{I}_{2}$ for known values of the Doppler shift can be calculated using curves fitted to each slope of the iodine absorption line. The un-shifted illumination frequencies are found from their initial transmissions, then the Doppler shift is then added to each frequency and the transmission at this shifted frequency can then be found. Finally the ratio $T_{1} / T_{2}\left(=I_{1} / I_{2}\right)$ for that value of the Doppler shift is calculated. The ratio of the measured intensities in the two images can then by used with the lookup table to find the Doppler shift. Using this approach the range of Doppler shifts is no longer restricted to just the linear portion of the iodine absorption line, allowing a greater range of flow velocities to be measured.

Both of the normal and increased sensitivity approaches overcome several problems identified with conventional PDV. In conventional PDV superposition of the two images to sub-pixel accuracy is essential if errors in the calculated velocities are to be minimized. For example Thorpe et al[13] assess the impact of image misalignment, on the velocity field of a rotating disc, for an image misalignment of 0.1 pixels, they give an estimate of this error as $\pm 5 \mathrm{~ms}^{-1}$. Errors due to poor image registration become particularly troublesome if large velocity gradients are present in the region imaged. The main causes of poor image registration are differences between the optical aberrations and magnifications of the two imaging paths so errors tend to be worse towards the outside edges of the images, where these factors are largest. In $2 v$-PDV alignment of the reference and signal images on the active area of the camera is no longer an issue and the signal/reference image misalignment problem found in conventional PDV is avoided.

The $2 v$-PDV method also eliminates the polarization sensitivity of the split ratio of the beam splitter used in two-camera systems[14]. Ideally the beam splitter used in a conventional PDV imaging head would split the incoming light 50:50 between the signal and reference cameras with no variation for different polarizations of light. However even the 'non-polarizing' beam splitters typically used retain a slight sensitivity to polarization, typically quoted as $\pm 3 \%$ variation in the split ratio for $\mathrm{S}$ and $\mathrm{P}$ polarized light, leading to typical velocity errors of $\pm 7 \mathrm{~ms}^{-1}$.

A two-wavelength method has been described by Arnette et al [15], using two laser lines (532 and 618nm) and a colour CCD to capture signal and reference images simultaneously. However the effects of different scattering efficiencies from the particles at the two widely spaced wavelengths were not taken into account, nor were the differing responses of the CCD used at these different wavelengths. The arrangement of pixels in a colour camera would imply that there is an image offset of at least one and possible two pixels between the signal and reference image, so image misalignment issues remain. Another problem was that of "signal bleed" of the scattered intensity from one pixel to another led that led to significant errors. It is also unclear what effect the absorption bands in the region of the $618 \mathrm{~nm}$ reference beam had on the reference signal, as the iodine absorption spectrum was not reported either experimentally or theoretically at this wavelength.

Muller et al [16] described a similar method using a frequency modulated Distributed Bragg Reflector (DBR) laser diode $(852.6 \mathrm{~nm}, 100 \mathrm{~mW})$ and a Caesium absorption cell to capture three sequential images at three wavelengths for a single velocity component.

\section{Multiple viewing directions using imaging fibre bundles}

Viewing the flow from a single observation direction allows a single component of the flow velocity to be measured; therefore to make three-dimensional measurements a minimum of three viewing directions are necessary. Conventional methods use a separate PDV imaging head (two cameras and an iodine cell each) to measure each of the three velocity components. The use of imaging fibre bundles, pioneered at Cranfield University $[9,17,18]$, to port multiple views of the region of interest to a single detector head, considerably simplifies the system and makes it possible to make three component velocity measurements using a single PDV imaging head. A similar multi-branch imaging fibre bundles has been described by Willert et al [19].

The custom made imaging fibre bundles in use at Cranfield uses a coherent array of fibres that is spilt into four channels (figure 5). Each channel is $4 \mathrm{~m}$ long, and has 600x500 fibres that are $8 \mu \mathrm{m}$ in diameter and positioned at $10 \mu \mathrm{m}$ centres. Cross talk between fibres is minimised by the use of interstitial absorbers between fibres[20]. The losses through the bundle are $\sim 50 \%$ for the $4 \mathrm{~m}$ length used. The four views are 
combined at the detector head, with each occupying a quarter of the CCD image (figure 6). An example of the image formed is shown in figure 7. This is a view of a calibration target used to de-warp the image to a common view and determine the observation directions for each view[21]. Figure 8 shows the result after this de-warping process; here all four views have been overlaid for demonstration purposes.



Figure 5. The individual arms of the imaging fibre bundles.

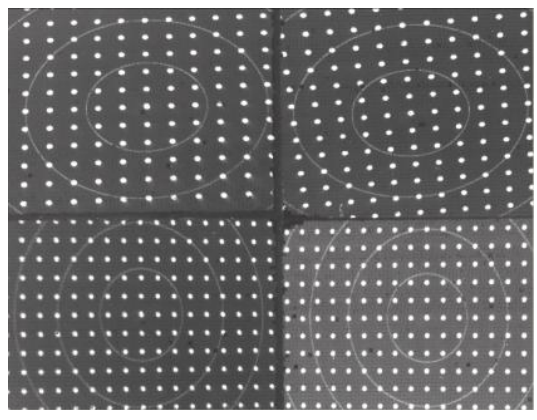

Figure 7. An example image of a view through the imaging bundles of a calibration target (field of view $\sim 100 \times 100 \mathrm{~mm}$ ) the white dots are $2.5 \mathrm{~mm}$ in diameter and spaced on a regular grid of $10 \mathrm{~mm}$.

\section{Experimental Arrangement}

\subsection{Illumination system}

In our previous work using 2v-PDV[10] a combination of acousto-optic modulators (AOMs) were used to shift the optical frequency of an argon-ion laser to provide two illumination frequencies separated by approximately $700 \mathrm{MHz}$. The light source used was a tuneable Argon-ion laser (Spectra Physics Beamlok 2060), incorporating a temperature-stabilized etalon to ensure single-mode operation at $514.5 \mathrm{~nm}$. The single-mode line width was about $2 \mathrm{MHz}$ and optical frequency stability was $\sim 10 \mathrm{MHz}$ jitter plus a long-term drift with ambient temperature of $\sim 50 \mathrm{MHzK}^{-1}$. This set-up allowed rapid switching between the two illumination frequencies. However this system proved to be light inefficient due principally to multiple passes through a high frequency AOM, resulting in low illumination powers. This combined with the use of the imaging fibre bundles, with an optical efficiency of approximately $50 \%$, resulted in low scattered light levels being collected on the CCD.

For this work the experimental arrangement was modified to allow greater illumination power; this new arrangement is shown in figure 9. The beam passes through an optical isolator to prevent back reflections entering the laser cavity; the polarisation is then adjusted using the first half wave plate to set the intensity of light entering the frequency locking system. The second half wave plate is used to adjust the polarisation for coupling into the high birefringent single mode polarisation maintaining fibre (York VSOP 2253-01F) used to guide the light to the beam scanning light sheet generator. 
Here the laser frequency is selected by changing the optical path length of the intra cavity etalon and is then stabilized using a locking system[22]. This consisted of an iodine cell, signal and reference photodiodes and locking electronics which adjusts the laser etalon temperature to ensure that the laser frequency is stable, based upon the transmission through this cell. The cell used in the locking system is a saturated cell, 100mm in length, which is held in a temperature controlled oven at $30^{\circ} \mathrm{C}$. It is necessary that the frequency of the locking beam is shifted by $260 \mathrm{MHz}$, using an acousto-optic modulator, so that the locking system can still operate if the laser frequency is tuned to $100 \%$ transmission. The locking beam will be shifted onto the absorption line so that any frequency fluctuations will result in an associated transmission fluctuation seen by the photodiodes and can then be corrected for. The addition of the locking system allowed the laser frequency stability to be improved to $\sim 3 \mathrm{MHz}$.

Although this arrangement increased the illumination power, the images are now captured with a separation of minutes rather than seconds, although for time average measurements presented here this has not proved to be a problem.

Both beams are coupled into the optical fibre and are then delivered to a prism-scanning device[6]. This scans the collimated beam rapidly across the region of interest, resulting in an ideal 'top-hat' intensity profile of the generated light sheet.

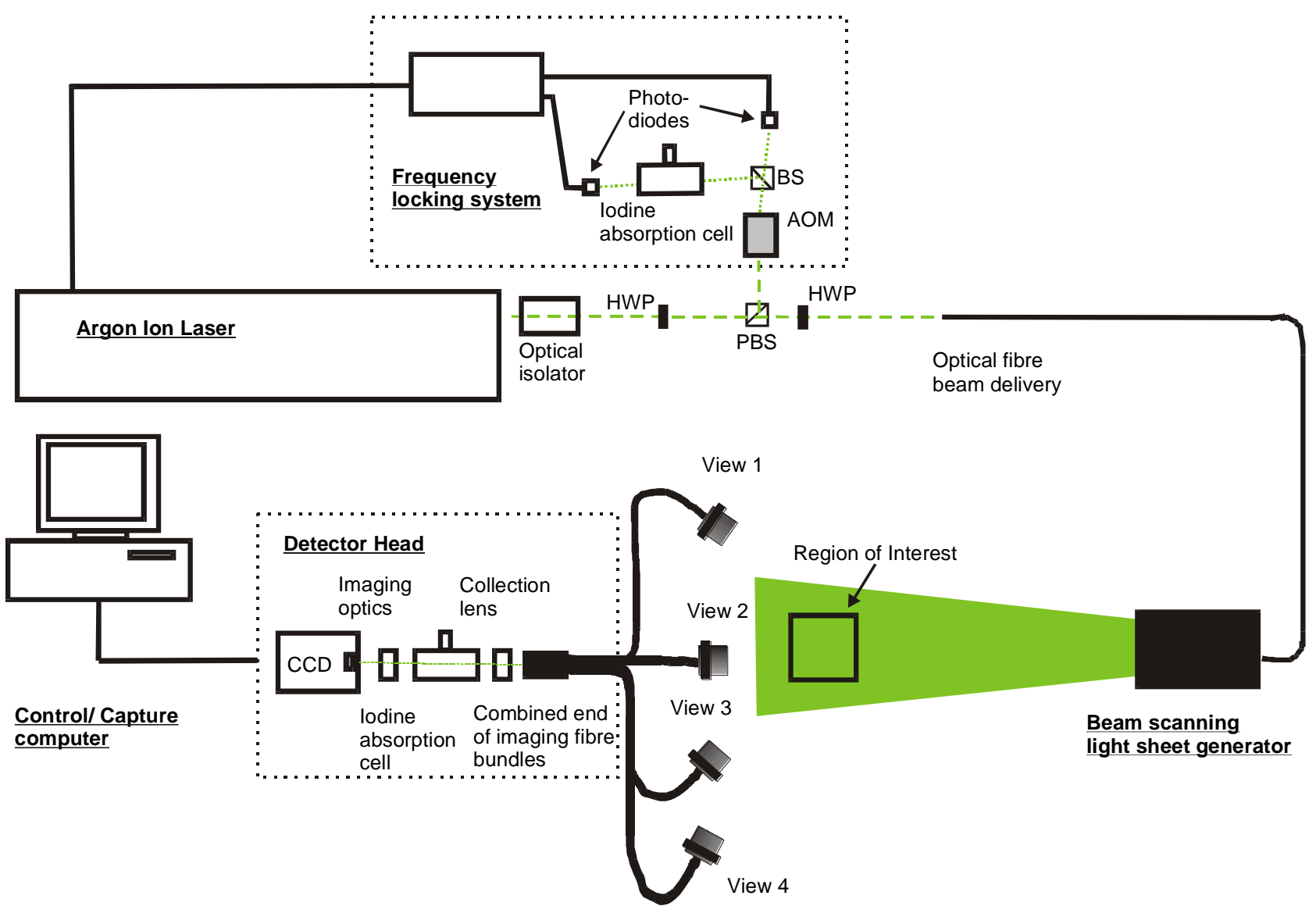

Figure 9. Schematic showing the experimental arrangement used for the three dimensional velocity measurements.

HWP - $\lambda / 2$ plate; AOM - acousto-optic modulator; PBS - polarising beam splitter; BS - beam splitter; HWP - half wave plate - Shifted (locking) beam path - - - - Un-shifted (illumination) beam

Previously when making single component measurements it was noted that a 'white card' type correction was needed[10] on the disc but not on a seeded flow measurement. This technique is used in conventional PDV to correct for differences in the optical paths for the two images. In our previous work a similar correction was thought to be necessary due to the differences in the spatial profile of the two beams exiting the optical 
fibre, used to transport them to the sheet generator. These differences were accentuated when a thick sheet was required; such as when illuminating the face of a disc, this resulted in the white card correction being necessary for these measurements. However with the arrangement described above both frequencies have identical paths so the spatial profile of both beams after the fibre will be identical avoiding the need for a white card type correction.

As well as providing increased beam powers, it is now possible to tune both illumination frequencies to coincide with either side of the absorption line allowing $2 v$-PDV measurements to be made using the second, increased sensitivity approach, described in section 2 . This was not possible using the combination of AOMs previously reported, as a frequency separation of greater than $700 \mathrm{MHz}$ is required to span the absorption line, and this was not achievable with the AOMs available.

\subsection{Image capture system.}

As described above the image capture system is greatly simplified by the use of imaging fibre bundles and the two-frequency technique, with the imaging head consisting of a single CCD camera and temperature stabilised iodine cell, figure 10, with the views being ported to the imaging head via the imaging fibre bundles. Each channel of the fibre bundles viewed the region of interest using 35mm SLR camera lenses coupled to the fibre using custom mounts. The light efficiency of the optical system is approximately 50\% through the imaging fibre bundles and 50\% from the output of the fibre bundles to the CCD. Although the numerical aperture of the imaging system is not well matched to the bundles the optics were chosen to produce high quality flat field images on the CCD chip.

The camera used was an 'Imager Intense' camera supplied by LaVision. This is a digital camera with 12 bit A/D conversion on a Peltier-cooled chip $\left(-15^{\circ} \mathrm{C}\right)$, with a 1376 by 1040 image resolution. The pixel size is $6.7 \mu \mathrm{m}$ by $6.7 \mu \mathrm{m}$. Dedicated image acquisition and processing software (DaVis) is used to control the camera and process and display the captured data. The integration time of the camera can be varied between $1 \mathrm{~ms}$ and 1000s, depending on the scattered light intensity. The camera dark current noise was found to be relatively constant with integration time, typically 40 counts, and can be removed using a background subtraction. Another important characteristic of the CCD is camera linearity. The manufacturer's specification quote a non-linearity of less than $1 \%$, this was previously investigated[10] and the camera found to be linear over $90 \%$ of full range. The remaining non-linear portion can be avoided by discarding pixel intensities over a certain threshold, typically $>3800$ counts.



Figure 10. Photograph showing the single camera imaging head arrangement.

The iodine cell, figure 11, which operates as a starved cell[23], is $25 \mathrm{~mm}$ in diameter and $50 \mathrm{~mm}$ long, and incorporates a cold finger that was used to determine the starvation temperature. The use of a starved cell means that the control of the cell temperature can be less stringent, as above the starvation temperature all 
the iodine is in vapour form, so the characteristics of the absorption will not vary greatly with temperature. The operating temperature should not be too much greater than the starvation temperature of the cell, otherwise thermal broadening of the absorption lines will alter the cell characteristics[24]. The starvation temperature of the cell used is $40^{\circ} \mathrm{C}$. The cold finger is held above this temperature using a Peltier element in a feedback loop, and the cell body is contained in an oven held at the same temperature $\left(50^{\circ} \mathrm{C}\right)$. The cell diameter is much smaller than those typically used in PDV $(50-100 \mathrm{~mm}[6,25])$, due to the use of the imaging fibre bundles, helping to maintain a constant temperature throughout the cell volume.

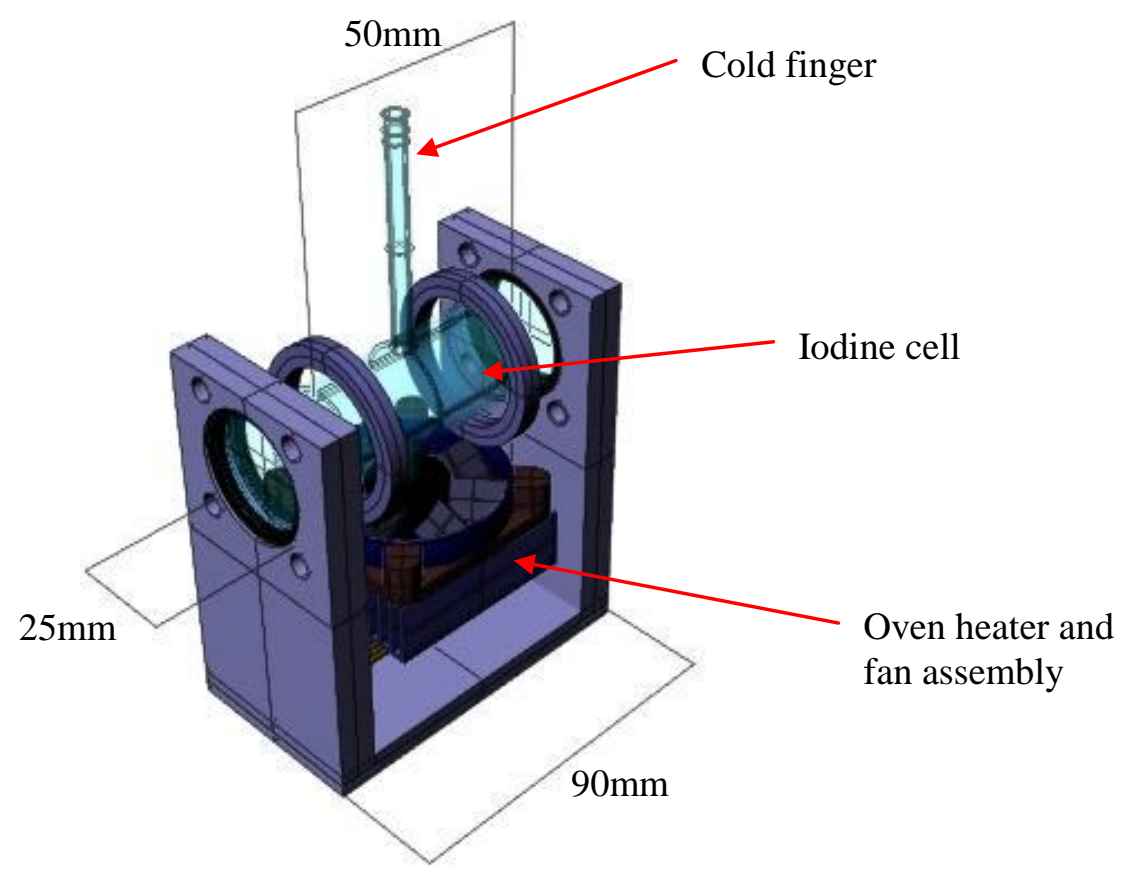

Figure 11. Schematic of the iodine cell and oven with covers removed

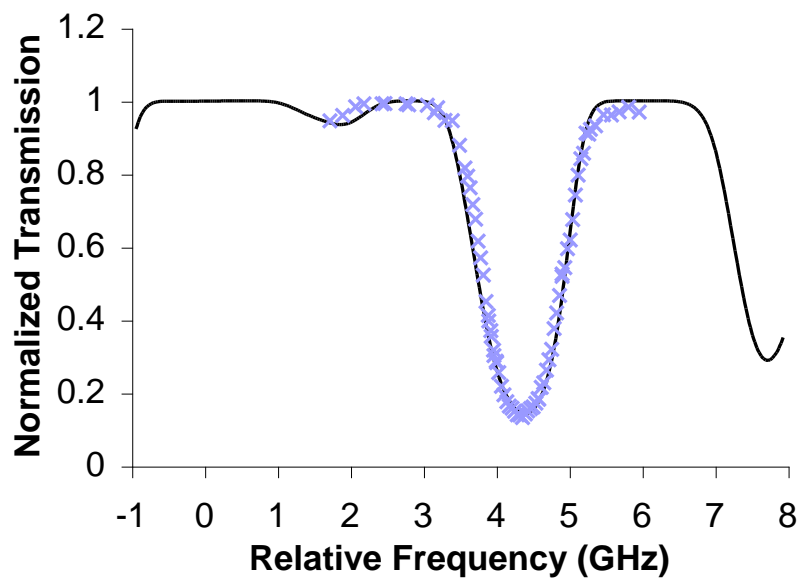

Figure 12. Experimental scan of the iodine absorption cell (crosses), shown plotted over the theoretical absorption spectrum[26].

In the first implementation of $2 v-P D V$, in which signal and reference images are captured, the shape of the iodine absorption around the $100 \%$ transmission region is of interest as the reference image is captured when the flow is illuminated with a frequency positioned here (figure 4, point A). As the reference image should be unaffected by Doppler shifts, this region needs to be flat, at $100 \%$ transmission, over the range of expected frequency shifts. Figure 12 shows an experimental scan of the iodine cell used in this work, overlaid on the theoretical iodine absorption, it can be seen that this region is flat over a range of $\sim 1 \mathrm{GHz}$. 
Slight differences exist between the modelled and measured transmission due to differences between the cell modelled and that used experimentally. Impurities in the cell as well as a slight difference in the quantity of iodine will both effect the transmission profile. In this work this is unimportant as the experimental curve was used in the calculations.

\subsection{Data collection / processing scheme.}

As the modified $2 v$-PDV system described above allowed sufficient frequency spacing between the two illumination frequencies it was possible to capture data using both the normal sensitivity and increased sensitivity approaches. Therefore to allow comparison between the two approaches, the data collection scheme consisted of capturing three images in succession under different illumination frequencies. The first image was captured when the laser was tuned, and locked to a full transmission position (figure 4, point A) next the laser was tuned to a point at approximately $50 \%$ transmission on the falling slope (figure 4, point B) and a second image captured. Finally the laser frequency was tuned to a similar position on the rising slope (figure 4, point $\mathrm{C}$,) and the third image captured. A background image was acquired before the data capture and is subtracted from all images to remove ambient scattered light and CCD dark current noise.

The first two images are analogous to the reference and signal images in conventional PDV and are used to produce a normalised transmission image in the normal sensitivity $2 v$-PDV approach. The second and third image can be used in the increased sensitivity $2 v$-PDV approach to produce a normalised transmission image as described in section 2 using (2)-(6).

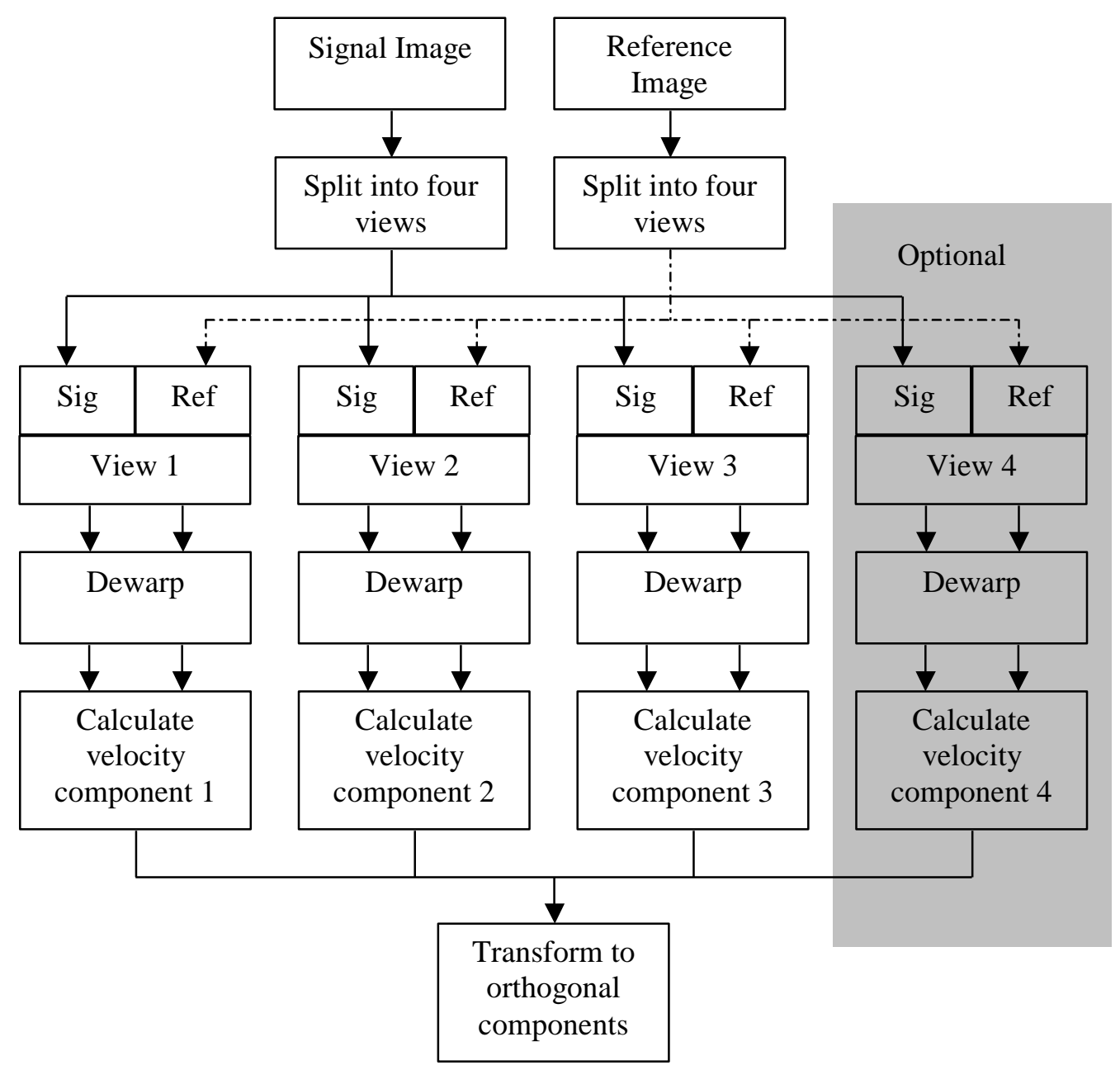

Figure 13. Flow diagram detailing the processing scheme for a 3D PDV measurement. 
An overview of the processing scheme used when making 3D measurements is shown in figure 13 . Before the normalised transmission is calculated the image is split into the four views and each view is de-warped to a common view. The de-warping process also calculates the observation direction for each view on a pixelby-pixel basis[21]. The de-warping process requires a target to be placed in the region of interest and an image captured. Software is then used to identify the markers on the target and map the image to a regular grid. A threshold is also applied to the images to remove pixels whose signal levels are too low or are saturated (typically signal levels of $<50$ counts and $>3800$ counts).

In conventional PDV processing it is necessary to apply a white card correction to the ratio image to obtain the normalised transmission image. The need for this correction in conventional PDV has also been observed in our previous work, as well as by other researchers[27-29]. However this is not necessary when using the $2 v$-PDV techniques due to the automatic alignment between the two images used.

Many researchers [27,28] also apply low pass filters to the data or processed results to remove high frequency noise, however in this work this was found to be unnecessary and so has been avoided or kept to a minimum. Finally once each view has been processed to a measured velocity component it is necessary to convert from these non-orthogonal components to the three orthogonal velocity components, $\mathrm{U}, \mathrm{V}$ and $\mathrm{W}$.

\section{Results}

\subsection{Three dimensional velocity measurements on a rotating disc.}

The system was used to measure the velocity field of a rotating disc. This provides a well-known velocity field with which to characterise the performance of the system. Each arm of the imaging fibre bundle was used with a $35 \mathrm{~mm}$ SLR camera lens to view the rotating disc. The disc itself was $200 \mathrm{~mm}$ in diameter, although the common field of view of each observation direction was an approximate disc $100 \mathrm{~mm}$ in diameter. The rotation of the disc was measured using an optical tachometer giving a maximum velocity in the field of view of $\sim \pm 34 \mathrm{~ms}^{-1}$. The geometry for the measurements is shown in figure 14 , with three views $\left(\mathrm{O}_{1}, \mathrm{O}_{2}\right.$ and $\left.\mathrm{O}_{4}\right)$ located in forward scatter and the third located in backscatter. Views 3 and $4\left(\mathrm{O}_{3}\right.$ and $\left.\mathrm{O}_{4}\right)$ are in the XZ plane and view 1 and $2\left(\mathrm{O}_{1}\right.$ and $\left.\mathrm{O}_{2}\right)$ are raised/lowered out of the plane.

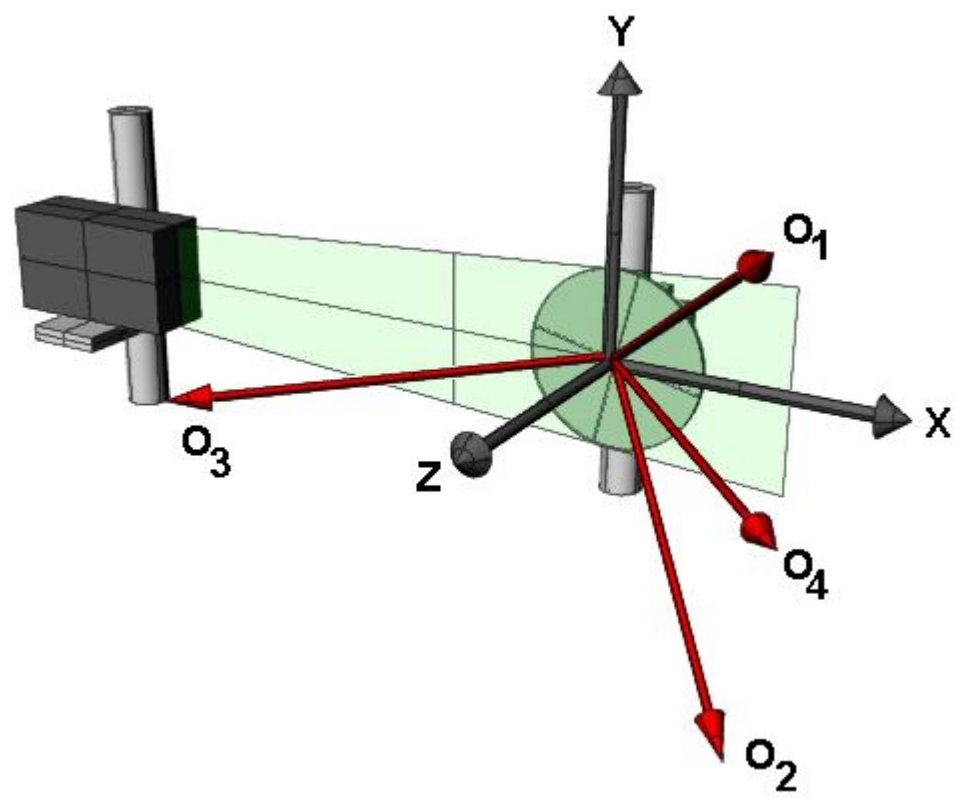

Figure 14. Diagram showing the viewing geometry used when making measurements on the rotating disc. $\mathrm{O} 1, \mathrm{O} 2, \mathrm{O} 3$ and $\mathrm{O} 4$ are the observation directions for each arm of the imaging fibre bundles.

Measurements were made using both $2 v$-PDV approaches described above. In order to measure all three components of velocity it is necessary to view from at least three directions, however the imaging fibre bundle in use has four channels so all four were used to view the face of the disc. The process of converting 
the measured velocity components to the three orthogonal components magnifies the error present. This results in the errors in the orthogonal components being greater than those in a single velocity component measurement. We have found that the use of a fourth measured velocity component can significantly reduce these conversion errors. The viewing configuration used for disc measurements is also far from ideal due to the restriction of only being able to view the light sheet (the face of the disc) from a single side, so this magnification is greater than it would be for real seeded flow experiments where the light sheet can be viewed from both sides. The use of additional velocity components and the optimum positioning for the viewing direction will be discussed in more detail in a forthcoming publication.

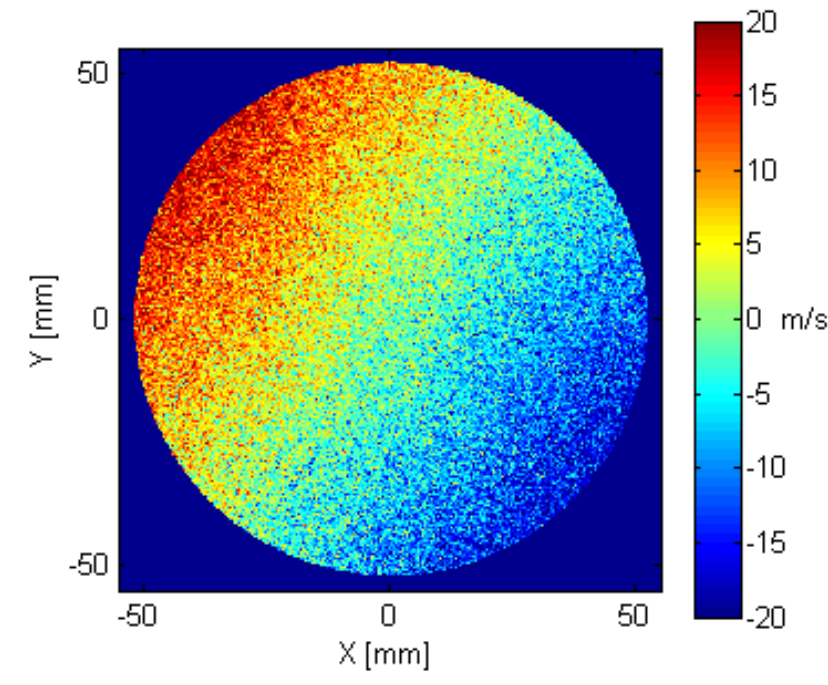

Figure 15. A single measured velocity component of a rotating disc, calculated using the normal sensitivity scheme.

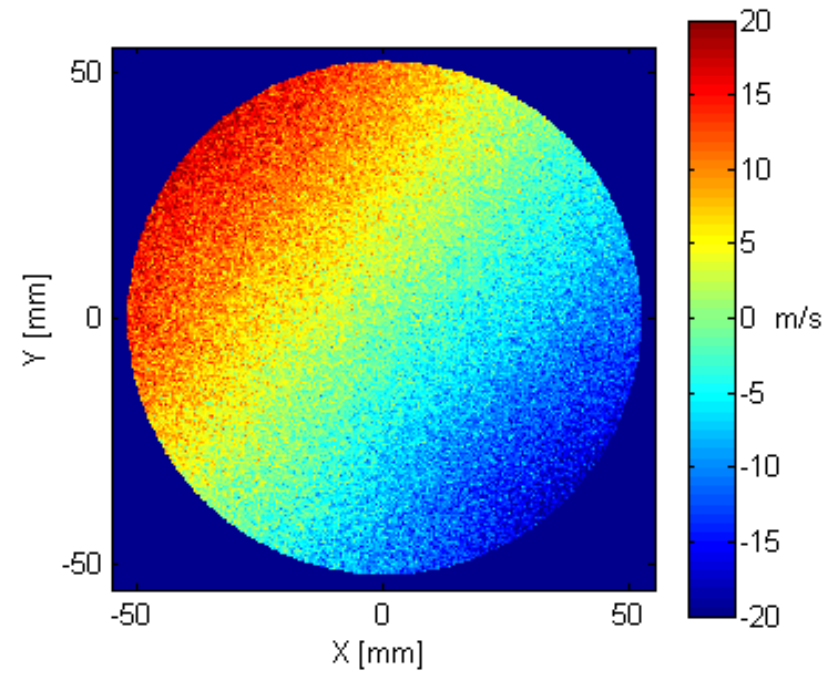

Figure 16. A single measured velocity component of a rotating disc, calculated using the increased sensitivity scheme.

Each observation direction was processed to yield the measured velocity component given by $(\hat{\boldsymbol{o}}-\hat{\boldsymbol{\imath}})$ for both the normal and increased sensitivity $2 v$-PDV schemes. As discussed above, both illumination frequencies beams have identical paths which results in identical signal and reference illumination profiles and there being no need to apply the 'white card' correction to realise the velocity field. The low-pass filtering commonly used in PDV processing was kept to a minimum, with no filtering at all applied to these results.

Figure 15 and figure 16 show examples of a single measured velocity component for both sensitivity schemes. Here it can be seen that the velocity component captured using the increased sensitivity scheme is visibly less noisy than that captured using the normal sensitivity scheme.

In order to assess the benefit of the increased sensitivity scheme, a theoretical velocity component was generated, for all points on the wheel, for each view. At each point this was subtracted from the calculated component and a histogram of this variation over the image plotted. Figure 17 shows the resulting histograms for a single velocity component for both normal and increased sensitivity schemes. Here it can be seen that the resulting error is smaller for the increased sensitivity scheme. 




Figure 17. Histograms showing the difference between the measured and theoretical velocity component.

+ Normal sensitivity $2 v$-PDV technique, ${ }^{*}$ Increased sensitivity $2 v$-PDV technique.

Similar plots were produced for all four velocity components and the standard deviations for these are shown in table 1 . It can be seen that there is approximately a $40 \%$ reduction in the level of error when using the increased sensitivity scheme over the normal sensitivity scheme.

Table 1. Standard deviations of the variation between the measured and theoretical velocity components, and the calculated reduction in error when using the increased sensitivity scheme.

\begin{tabular}{lcccc}
\hline Standard deviation & View 1 & View 2 & View 3 & View 4 \\
\hline Normal sensitivity & $4.6 \mathrm{~ms}^{-1}$ & $4.9 \mathrm{~ms}^{-1}$ & $2.4 \mathrm{~ms}^{-1}$ & $3.9 \mathrm{~ms}^{-1}$ \\
Increased sensitivity & $2.5 \mathrm{~ms}^{-1}$ & $2.9 \mathrm{~ms}^{-1}$ & $1.6 \mathrm{~ms}^{-1}$ & $2.3 \mathrm{~ms}^{-1}$ \\
\% Reduction & $45.7 \%$ & $40.8 \%$ & $33.3 \%$ & $41.0 \%$ \\
\hline
\end{tabular}

The measured velocity components are then converted to the orthogonal velocity components $\mathrm{U}, \mathrm{V}$ and $\mathrm{W}$, representing the vertical, horizontal and out-of-plane components. Figure 18 shows a comparison between the two sensitivity schemes for these orthogonal velocity components. The improved sensitivity can also be seen in these calculated orthogonal components with the increased sensitivity results being visibly smoother. Again if these calculated orthogonal components are compared with the theoretical orthogonal velocity components and histograms taken of this variation, the increased sensitivity scheme makes a considerable improvement. The standard deviations of this, shown in table 2, are again typically $40 \%$ smaller for the increased sensitivity scheme compared with the normal sensitivity scheme, as would be expected from the improvement in the measured velocity components 


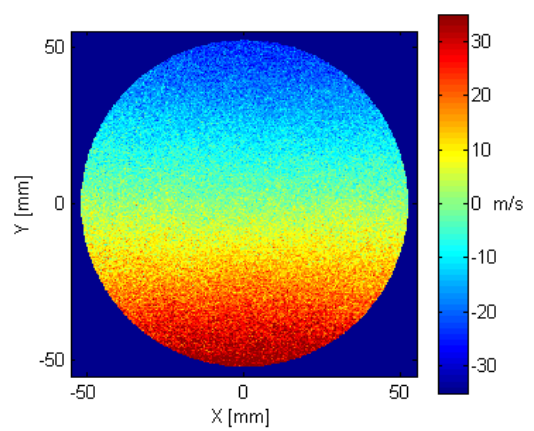

U (normal sensitivity)

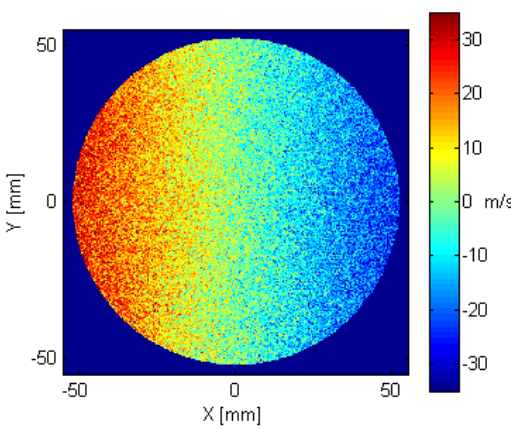

V (normal sensitivity)

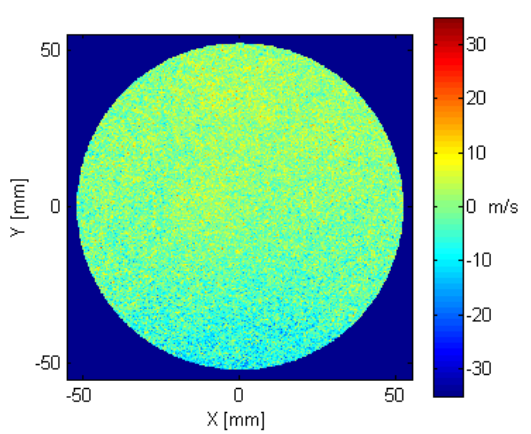

W (normal sensitivity)

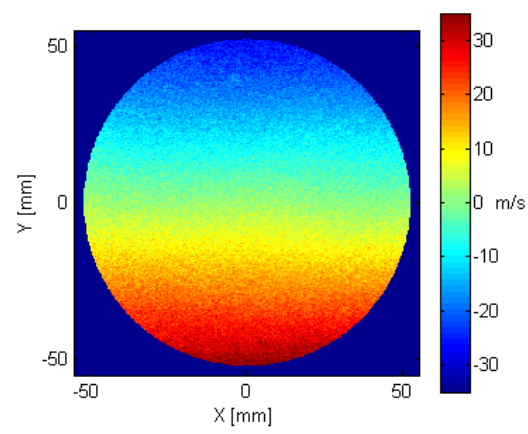

U (increased sensitivity)

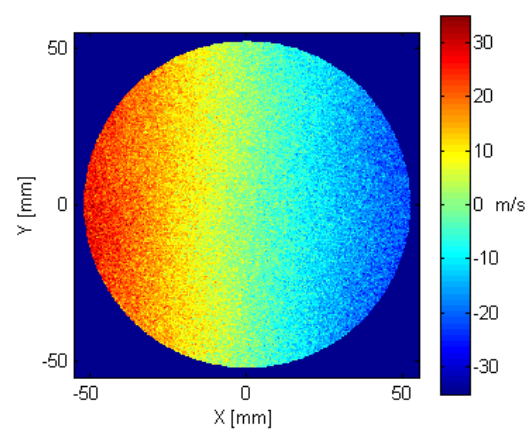

V (increased sensitivity)

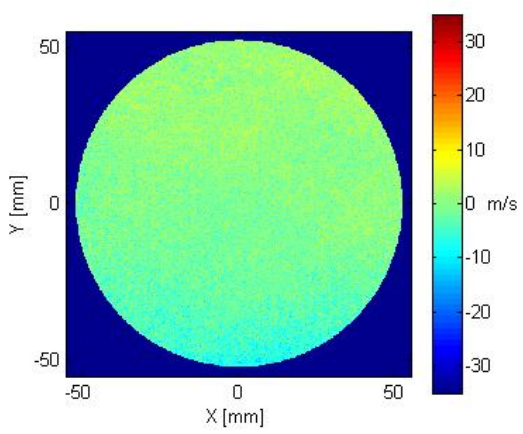

W (increased sensitivity)



$\mathrm{U}$ error

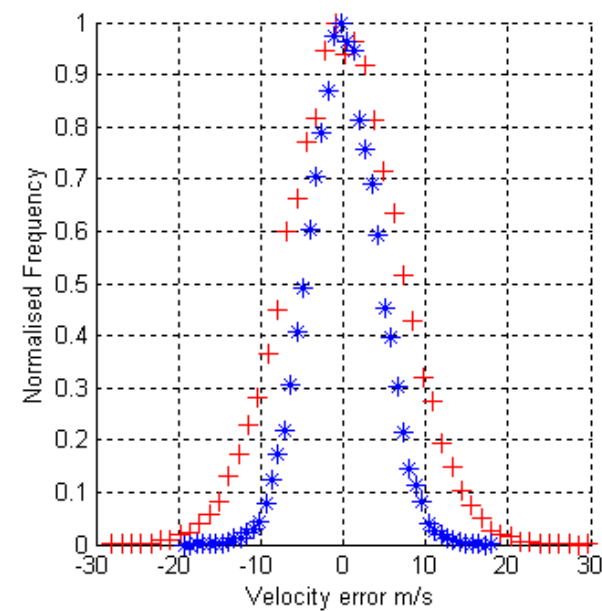

V error



W error

Figure 18. Comparison of computed orthogonal velocity components; (left column) Examples of the calculated orthogonal velocity components using the normal sensitivity $2 v$-PDV technique; (middle column) Examples of the calculated orthogonal velocity components using the increased sensitivity $2 v$-PDV technique; (right column) Histograms showing the difference between the measured and theoretical velocity component. + Normal sensitivity $2 v$-PDV technique. * Increased sensitivity $2 v$-PDV technique. 
Table 2. Standard deviations of the variation between the calculated and theoretical orthogonal velocity components, and the calculated reduction in error when using the increased sensitivity scheme.

\begin{tabular}{lccc}
\hline Standard deviation & $\mathrm{U}$ & $\mathrm{V}$ & $\mathrm{W}$ \\
\hline Normal sensitivity & & & $4.4 \mathrm{~ms}^{-1}$ \\
Increased sensitivity & $3.6 \mathrm{~ms}^{-1}$ & $6.8 \mathrm{~ms}^{-1}$ & $2.4 \mathrm{~ms}^{-1}$ \\
\% Reduction & $2.2 \mathrm{~ms}^{-1}$ & $4.2 \mathrm{~ms}^{-1}$ & $38.2 \%$ \\
\hline
\end{tabular}

\subsection{Three dimensional velocity measurements on a seeded air jet}

The system was then demonstrated on an axis-symmetric air jet, with a $20 \mathrm{~mm}$ diameter smooth contraction nozzle that was seeded using a smoke generator producing particles in the $0.2-0.3 \mu \mathrm{m}$ diameter range. The jet has a theoretical exit velocity of $94 \mathrm{~ms}^{-1}$, which was calculated by measuring the nozzle pressure ratio. Images where captured with an integration time of 10 seconds.

The system was set-up as shown in figure 19, with three views positioned on one side of the sheet and the fourth viewing from the opposite side. As mentioned above the ability to position views on opposite sides of the light sheet greatly improves the propagation of errors from the measured velocity components to the orthogonal velocity components. An example of a measurement is shown in figure 20, with vectors showing the in-plane velocities and the colour representing the out-of-plane component.



Figure 19. 3D schematic diagram showing the experimental configuration used for measurements on the seeded air jet.

As the jet was seeded through the air intake, seeding levels outside of the jet were low resulting in low levels of scattered light for these regions. As any velocity measurements for these regions would be unreliable a threshold has been applied to the data. As with the disc measurements four viewing directions, and hence four velocity components, were used in the calculation of the orthogonal velocity components to minimise the final errors. Similarly no 'white-card' correction was necessary and low pass filtering was minimal, a single pass of a $3 \times 3$ averaging filter in order to smooth the vectors. It can be seen that the measurements show the expected development of the jet, in the main flow direction, with minimal out-of-plane velocities. 


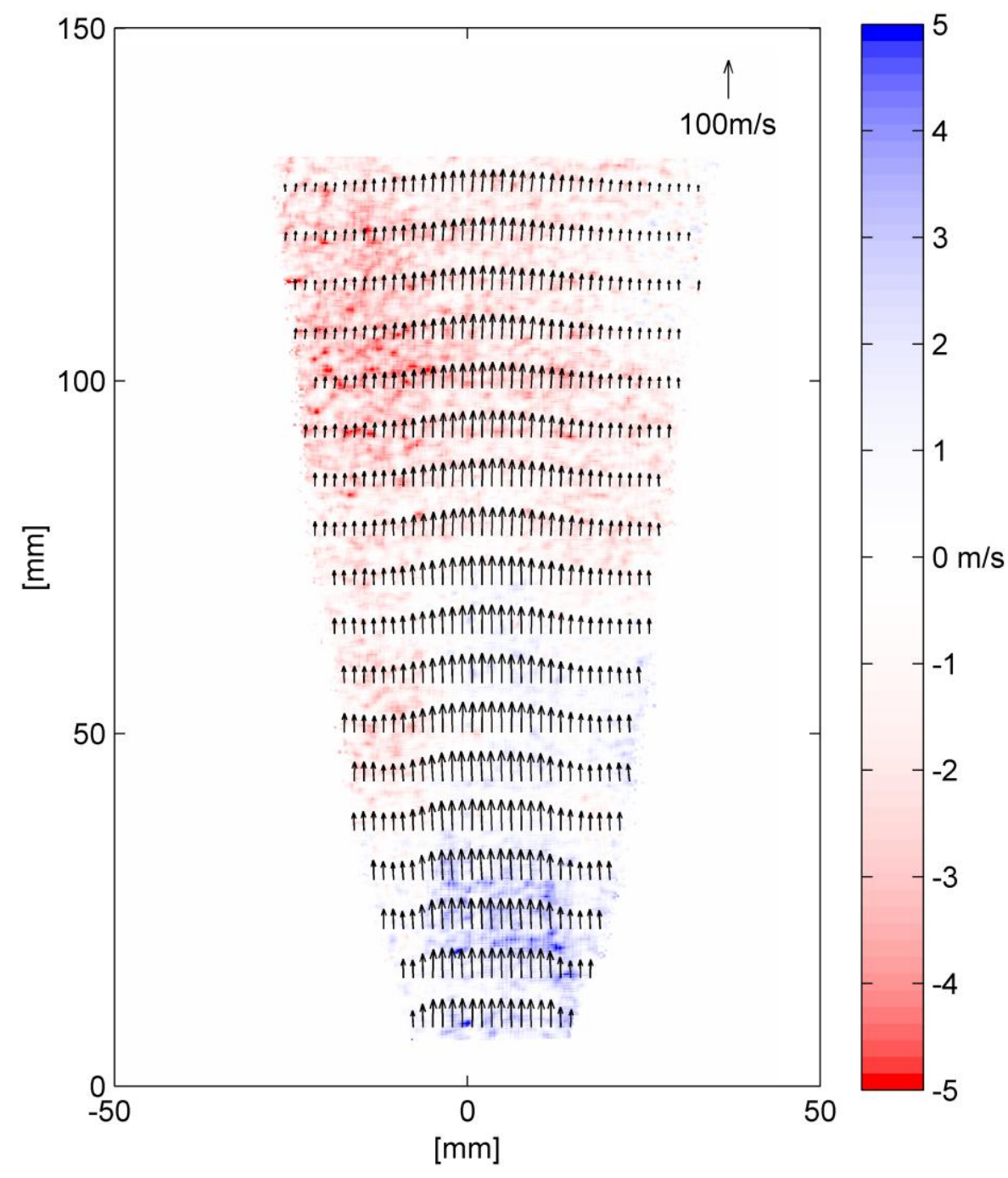

Figure 20. Example of a 3D 2v-PDV measurement made on a seeded air jet. Vectors show the in plane velocities (every $8^{\text {th }}$ and $40^{\text {th }}$ vector show in the horizontal and vertical directions respectively) and colour the out of plane velocity.

A second set of measurements were then made with the jet nozzle rotated, so that the main flow was in the out-of-plane direction. A 'swirler' was introduced into the nozzle, in an attempt to create more in-plane flow velocities. The nozzle was positioned at various distances from the sheet allowing the measurement of the flow velocities in several slices through the jet; this is shown in figure 21 . A single slice, at $120 \mathrm{~mm}$ downstream from the nozzle, is shown in figure 22. In these measurements the vectors represent the in-plane velocity components and the colour represents the out-of-plane velocity component. Again seeding was introduced to the jet via the air intake, so signal levels in regions outside of the jet were low so measurements in these regions have been removed using a threshold. It can be seen, in figure 22, that the jet was swirling and drawing in air predominantly from one side of the flow, this could also been seen visually in the light sheet.

\section{Discussion and Conclusions}

A PDV technique using two illumination frequencies and imaging fibre bundles has been demonstrated that is capable of measuring all three components of velocity, across a plane defined by a light sheet, using a 
single CCD camera. As an extension of our previous work, where single component, time averaged, velocity measurements were made, the system was expanded to measure all three components of velocity using imaging fibre bundles. The system was demonstrated with 3D velocity measurements on a rotating disc and a seeded air jet.



Figure 21. 3D $2 v$-PDV cross section measurements on a seeded air jet. Vectors represent the in plane velocities $\left(\right.$ every $12^{\text {th }} \times 12^{\text {th }}$ vector shown) and colour the out-of-plane velocity. Cross sections were measured at 60,80,100 and 120mm distances from the jet nozzle exit. 



Figure 22. 3D 2v-PDV cross section measurements on a seeded air jet at $120 \mathrm{~mm}$ downstream from the nozzle exit. Vectors represent the in plane velocities (every $12^{\text {th }} \times 12^{\text {th }}$ vector shown) and colour the out-of-plane velocity.

The combination of the $2 v$-PDV technique with the imaging fibre bundles allows 3D velocity measurements to be made using only a single camera and iodine cell, greatly reducing the cost and complexity associated with conventional PDV techniques which require six cameras and three iodine cells for 3D measurements. The use of the imaging fibre bundles also allows greater flexibility in the positioning of the observation directions.

As both signal and reference images are captured on a single camera the pixel-matching problem is overcome resulting in there being no need to use a 'white card' type correction that is commonly applied in PDV measurements. However the images used in the normalisation process are now captured sequentially, unlike in conventional PDV where they are captured simultaneously. For steady-state flows this should not be a problem, provided that the seeding is relatively dense and the seeding distribution remains essentially unchanged during the time taken to acquire the two images.

In a rapidly time-varying flow, averaging over long integration times is not an option; a pulsed laser would be required for this type of flow, to freeze the motion of the particles. An investigation of this is currently under way.

The ability to further improve the sensitivity of the two-frequency PDV technique, by positioning both illumination frequencies on opposite sides of the transfer function, has been demonstrated. By comparison with the theoretical velocity field for a rotating disc a typical reduction in the error of approximately $40 \%$ can be achieved using the increased sensitivity scheme. 


\section{Acknowledgement}

This work was funded by the Engineering and Physical Sciences Research Council (EPSRC), UK (GR/SO4291).

\section{References}

[1] Samimy, M. and Wernet, M.P., 2000, Review of Planar Multiple-Component Velocimetry in HighSpeed Flows, AIAA Journal, 38, 4, 553-574.

[2] Komine, H., Brosnan, S., Litton, A., and Staepperts, E., 1991, Real-Time Doppler Global Velocimetry, AIAA 29th Aerospace Sciences Meeting, Reno, Nevada, Paper 91-0337.

[3] Meyers, J.F. and Lee, J.W., 1991, Proof of concept test of the Doppler Global Velocimeter, Spaceborne Photonics: Aerospace Applications Of Lasers And Electro-optics, Newport Beach, CA,

[4] Meyers, J.F., Lee, J.W., and Schwartz, R.J., 2001, Characterization of Measurement Error Sources in Doppler Global Velocimetry, Measurement Science and Technology, 12, 357-368.

[5] Ford, H.D. and Tatam, R.P., 1997, Development of Extended Field Doppler Velocimetry for Turbomachinery Applications, Optics and Lasers in Engineering, 27, 675-696.

[6] Roehle, I., Willert, C., Schodl, R., and Voigt, P., 2000, Recent Developments and Applications of Quantitative Laser Light Sheet Measuring Techniques in Turbo machinery Components, Measurement Science and Technology, 11, 1023-1035.

[7] Ford, H.D., Nobes, D.S., and Tatam, R.P., 2001, Acousto-optic Frequency switching for single-camera planar Doppler Velocimetry, SPIE proceedings, Optical Diagnostics for Fluids, Solids and Combustion, Eds. C.R. Mercer, S.S. Cha and G. Shen, San Diego, CA, Vol. 4448, pp 272-282.

[8] Roehle, I. and Willert, C., 2001, Extension of Doppler Global Velocimetry to Periodic Flows, Measurement Science and Technology, 12, 420-431.

[9] Nobes, D.S., Ford, H.D., and Tatam, R.P., 2004, Three Component Planar Doppler Velocimetry Using Imaging Fibre Bundles, Experiments in Fluids, 36, 1, 3-10.

[10] Charrett, T.O.H., Ford, H.D., Nobes, D.S., and Tatam, R.P., 2004, Two frequency Planar Doppler Velocimetry (2v-PDV), Review of Scientific Instruments, 75, 11, 4487-4496.

[11] Fischer, M., Heinze, J., Matthias, K., and Roehle, I., 2000, Doppler Global Velocimetry in Flames Using a newly Developed, Frequency Stabilized Tunable, Long Pulse Nd:YAG Laser, 11th International Symposium on Applications of Laser techniques to Fluid Mechanics, Lisbon, Portugal,

[12] Gerstenkorn, S. and Luc, P., 1986, Atlas du Spectre d'Absorption de la Molecule d'Iode 14800-200 cm ${ }^{1}$ Complement: Identification des Transitions du Systeme $(B-X)$, Paris, France, Editions du Centre Nationale de la Recherche Scientifique,

[13] Thorpe, S.J., Ainsworth, R.W., and Manners, R.J., 1995, The Development of a Doppler Global Velocimeter and its Application to a Free Jet Flow, ASME / JSME Fluids Engineering and Laser Anemometry Conference and Exhibition, Hilton Head, SC, USA,

[14] Meyers, J.F. and Lee, J.W., 2000, Identification and Minimization of Errors in Doppler Global Velocimetry Measurements, 10th International Symposium on Applications of Laser techniques to Fluid Mechanics, Lisbon, Portugal,

[15] Arnette, S.A., Elliott, G.S., and Mosedale, A.D., 2000, Two-colour planar Doppler Velocimetry, AIAA Journal, 38, 11, 2001-2006.

[16] Muller, H., Eggert, M., Pape, N., Dopheide, D., Czarske, J., Buttner, L., and Razik, T., 2004, Time resolved DGV based on laser frequency modulation, 12th International symposium on the application of Laser Techniques to fluids, Lisbon, Portugal.

[17] Nobes, D.S., Ford, H.D., and Tatam, R.P., 2001, Instantaneous, Two Camera, Three Dimensional Planar Doppler Velocimetry using Imaging Fiber Bundles, SPIE Proceedings, Optical Diagnostics for Fluids, Solids and Combustion, San Diego, Vol. 4448, pp 72-83.

[18] Nobes, D.S., Ford, H.D., and Tatam, R.P., 2003, Planar Doppler Velocimetry Measurements of Flows using Imaging Fiber Bundles, Proc. SPIE, 5191, pp 122-133.

[19] Willert, C., Stockhausen, G., Beversdorff, M., klinner, J., Lempereur, C., Barricau, P., Quest, J., and Jansen, U., 2005, Application of Doppler Glbal velocimetry in cryogenic wind tunnels, Experiments in Fluids, 39, 420-430. 
[20] Schott AG website, 2005, http://www.schott.com

[21] Nobes, D.S., Wieneke, B., and Tatam, R.P., 2004, Determination of View Vectors from Image Warping Mapping Functions, Optical Engineering, 43, 2, 407-414.

[22] Chehura, E. and Tatam, R.P., 2003, In-line Laser Doppler Velocimeter using fibre-optic Bragg grating interferometric filters, Measurement Science and Technology, 14, 724-735.

[23] Quinn, T.J. and Chartier, J.-M., 1993, A New Type of Iodine Cell for Stabilized Lasers, IEEE Transactions on Instrumentation and Measurement, 42, 405-406.

[24] Chan, V.S.S., Heyes, A.L., Robinson, D.I., and Turner, J.T., 1995, Iodine Absorption Filters for Doppler Global Velocimetry, Measurement Science and Technology, 6, 784-794.

[25] Reinath, M.S., 2001, Doppler Global Velocimeter Development for Large Wind Tunnels, Measurement Science and Technology, 12, 432-441.

[26] Forkey, J.N., 1996, Development and Demonstration of Filtered Rayleigh Scattering - a Laser Based Flow Diagnostic for Planar Measurements of Velocity, Temperature and Pressure, Final Technical Report for NASA Graduate Student Researcher, Fellowship Grant \#NGT-50826, Princeton University.

[27] Reinath, M.S., 1997, Doppler Global Velocimeter Development for the Large Wind Tunnels at Ames Research Center, NASA Technical Memorandum, 112210.

[28] Elliott, G.S. and Beutner, T.J., 1999, Molecular Filter Based Planar Doppler Velocimetry, Progress in Aerospace Sciences, 35, 799-845.

[29] McKenzie, R.L. and Reinath, M.S., 2000, Planar Doppler Velocimetry Capabilities at Low Speeds and its Application to a Full-Scale Rotor Flow (Invited), AIAA 21 st Aerodynamic Measurement Technology and Ground Testing Conference, Denver, Colorado, Paper 2000-2292. 